\title{
Construção da Hospitalidade através da Gastronomia Regional, Caso da comunidade indígena de Chacala, Município de Cuautitlán de García Barragán, Jalisco, México.
}

\author{
Construction of Hospitality through Regional Gastronomy, Case of the indigenous community of Chacala, \\ Municipality of Cuautitlán de García Barragán, Jalisco, Mexico
}

\author{
Fabiola Bobón Alvarado \\ Marisa Gutiérrez Estrada
}

Alfonso Zepeda Arce

Universidade de Santa Cruz do Sul - UNISC - Santa Cruz do Sul - Rio Grande do Sul - Brasil

\begin{abstract}
Resumo: A comida é uma referência permanente na memória quando se trata de recordar as experiências da viagem no tempo, a culinária está relacionada à história, ao que somos e ao que pertencemos, isto é, à nossa identidade. MINTZ (2003: 28) aponta que comer não é uma atividade puramente biológica porque "os alimentos ingeridos têm histórias associadas ao passado daqueles que as comem, as técnicas usadas para encontrar, processar, preparar, servir e consumir aqueles Os alimentos variam culturalmente e têm suas próprias histórias, e nunca são simplesmente consumidos, seu consumo é condicionado pelo significado ... "No caso do México, a culinária tradicional é um modelo cultural completo que inclui atividades agrárias, práticas rituais, conhecimento prático. técnicas e costumes antigos, culinários e comportamento comunitário ancestral, outro aspecto essencial no turismo é a hospitalidade, entendida como "o ato humano, exercido em um contexto doméstico, público e profissional, de receber, acolher, alimentar e entreter pessoas temporariamente deslocadas de seu habitat "(CAMARGO, 2003: 19), enquanto LASHLEY (2004; 2) conceitua o hospital Como um conjunto de atividades no setor de serviços relacionadas à oferta de alimentos, bebidas e / ou alojamento, ambos os elementos são baseados na cultura. No caso da comunidade indígena de Chacala, município de Cuautitlán, García Barragán, Jalisco, México propõe construir parte de sua hospitalidade baseada na culinária regional através da participação de membros da comunidade e da reavaliação do produção e preparação de alimentos tradicionais.
\end{abstract}

Palavras-chaves: Gastronomia, hospitalidade, comunidade indígena

Abstract: Food is a permanent reference in memory when it comes to remembering the experiences of time travel, cooking is related to history, to what we are and to what we belong, that is, to our identity. MINTZ (2003: 28) points out that eating is not a purely biological activity because the "... foods eaten have stories associated with the past of those who eat them, the techniques used to find, process, prepare, serve and consume those Foods vary culturally and have their own stories, and they are never simply eaten, their consumption is conditioned by the meaning ... "In the case of Mexico, traditional cuisine is a complete cultural model that includes agrarian activities, ritual practices, practical knowledge old, culinary techniques and customs and ancestral community behavior, another essential aspect in tourism is hospitality, understood as "the human act, exercised in a domestic, public and professional context, to receive, host, feed and entertain people temporarily displaced from their habitat "(CAMARGO, 2003: 19), meanwhile LASHLEY (2004; 2) conceptualizes the hospital ad as a set of activities in the services sector related to the offer of food, 
beverages and / or lodging, both elements are based on culture. In the case of the indigenous community of Chacala, municipality of Cuautitlán, García Barragán, Jalisco, Mexico proposes to build part of its hospitality based on regional cuisine through the participation of community members and the revaluation of the production and preparation of traditional foods.

Keywords: Gastronomy, hospitality, indigenous community

\section{Introducción}

La comida constituye un referente permanente en la memoria cuando se trata de recordar las experiencias de viaje en el tiempo (TORRES, 2003:305), jugando un papel importante en el marco de la economía de la experiencia contemporánea que significa el turismo (OCDE, 2012: 16) además de fijarse como parte esencial de la hospitalidad de un destino. De acuerdo a diversos estudios la cuota de gasto que los turistas dedican a la comida equivale a un tercio de su gasto total, significándose en un segmento sumamente atractivo para cualquier destino turístico (HERNÁNDEZ, 2015, FOX, 2007; HALL y SHARPLES, 2003; TELFER y WALL, 2000). En este contexto los ingresos por turismo internacional en el año 2017 alcanzaron la cifra de 1,34 billones de dólares de los EE: UU (OMT, 2018: 6).

Bajo esta perspectiva se puede afirmar que la gastronomía es un elemento esencial en el desarrollo de la actividad turística, pero para entender el tamaño y la complejidad que esta significa se debe en primera instancia definirla, para ello se tomó en cuenta el estudio realizado por Klynveld Peat Marwick Goerdeler (KPMG) titulado "La gastronomía en la economía española: Impacto económico de los sectores asociados", asumiendo como punto de partida que la gastronomía es comer y, por lo tanto equivale a la suma de un conjunto de actividades, que comienzan con la producción agrícola, ganadera y pesquera (con el comercio tanto interior, como exterior) siguiéndole, la industria de la alimentación y bebidas (procesados y no procesados), continuado en la distribución vía centrales de abastos, mercados, supermercados y desembocando en la comida y en la hostelería, es decir, es una suma de actividades que se resumen en el sector gastronómico (KGMG, 2019; 5-6 ). Este sector de acuerdo a KIVELA y CROTTS (2006: 354-
355) es un factor importante en el desarrollo de un turismo de nicho y de destinos especializados.

Por otra parte, se puede definir la hospitalidad como "el acto humano, ejercido en un contexto doméstico, público y profesional, de recibir, hospedar y alimentar y entretener a las personas temporalmente desplazadas de su hábitat" (CAMARGO, 2003:19), asimismo LASHLEY (2004: 2) conceptualiza la hospitalidad como un conjunto de actividades del sector servicios relacionadas con la oferta de alimentos, bebidas y/o hospedaje. Sin embargo, estos dos elementos clave de la actividad turística (gastronomía y la hospitalidad) comparten un respaldo que brinda en gran medida sus cimientos $y$ autenticidad, nos referimos a la cultura, por una parte la gastronomía es una faceta de la cultura de un país, que permite descubrir usos y costumbres de una región (ROCHAT, 2001, citado en PÉREZ, 2012: 3) y la hospitalidad como un rasgo cultural de los individuos.

En el caso de México los servicios de alojamiento temporal y de preparación de alimentos y bebidas se clasifican en el sector 72 , el cual contempla 568, 866 unidades económicas, de las cuales en el año 2013 de acuerdo al Censo Económico del INEGI (2014) el 97\% pertenecen al subsector de servicios de preparación de alimentos y bebidas, representando empleo al 6.6\% del personal ocupado en el país y una participación de 1.0\% del Producto Interno Bruto (PIB) de México.

Por otra parte, la asamblea de la UNESCO celebrada en Nairobi el 16 de noviembre de 2010 emitió la declaratoria formal de la cocina mexicana como patrimonio cultural de la humanidad, entendiendo que la cocina tradicional mexicana es un modelo cultural completo que comprende actividades agrarias, prácticas rituales, conocimientos prácticos antiguos, técnicas culinarias y costumbres y modos de 
comportamiento comunitarios ancestrales (UNESCO, $\mathrm{S} / \mathrm{F} ; 1)$.

En el caso particular del municipio de Cuautitlán de García de Barragán, ubicado en el estado de Jalisco, México son reconocidas las comunidades indígenas de Ayotitlán, Cuzalapa, Chacala, Plan de Méndez y Telcruz (CORRAL, 2015), descendientes de los nahuas que habitaron esta región antes de la Conquista española, y las cuales son caracterizadas por altos índices de marginación y una gran riqueza biocultural. De acuerdo con TETREAULT Y LÓPEZ (2001; 169) a pesar de los procesos de aculturación vividos, se mantienen rasgos culturales manifestados en sus ceremonias, sistemas de producción, estructuras sociales, creencias, tradiciones y forma de vida en general.

El municipo de Cuautiltán, colinda al norte con Casimiro Castillo, Autlán de Navarro y Tuxcacuesco, al este con Tuxcacuesco, Tolimán y el estado de Colima; al sur con el estado de Colima y el municipio de Cihuatlán; al oeste con los municipios de Cihuatlán, La Huerta y Casimiro Castillo. Tiene una población total de 18,923 habitantes y cuenta con una superficie de $1,361 \mathrm{~km} 2$, de éste el $60.7 \%$ tiene terreno montañoso y la mayor parte del municipio tiene clima cálido subhúmedo, las actividades a las que se dedican son industria, agricultura, comercio y servicios. Las comunidades indígenas, que ahí habitan, son quienes se encargan de conservar los recursos naturales y culturales pertinentes a Sierra de Manantlán (LOS MUNICIPIOS DE JALISCO, 2019:1).

\section{La Comunidad Indígena de Chacala}

La comunidad se localiza en las coordenadas GPS: Longitud (dec): -104.283889 Latitud (dec): 19.326667, a una mediana altura de 400 metros sobre el nivel del mar.

En relación a su historia existe poca información, solo aparece en el documento la Relación Geográfica de Cuzalapa y principalmente en el mapa de Cuzalapa fechado en 1531 junto a Cuzalapa y Ayotitlán, de acuerdo con el profesor Abel Vargas (2015: 17) su nombre nos lleva inmediatamente a pensar en la abundancia de "camarones del río" o langostinos que en la región lo llaman "chacales".

En la Relación Geográfica de Cuzalapa, se dice que en ese tiempo (1579) Chacala estaba sujeta a ella y haciendo referencia a la población, ese documento de 1579 dice textualmente: “...tiene otro sujeto Ilamado Chacala, que quiere decir Chacali porque, por junto al dicho pueblo pasa un río en el cual cogen muchos camarones colorados grandes, a los cuales Ilaman Chacales y, por esta razón, pusieron el dicho pueblo el dicho nombre de Chacala..." por estas noticias podemos concluir que el nombre de esta comunidad proviene del idioma náhuatl o "mexicano" y que significa "lugar de camarones de río" o "lugares de chacales", (VARGAS, 2015: 10).

Chacala tiene 1,024 habitantes distribuidos en en las distintas localidades que pertenecen a esta comunidad (Sehuaya, La Iguana, El Chico, La piedra, El arrayan, Mojoneras, Rancho Viejo, Terreros del chico, Chacala, Jocotlan y Picachos). 
Mapa 1: Localización del ejido de Chacala dentro de municipio de Cuautitlán de García Barragán

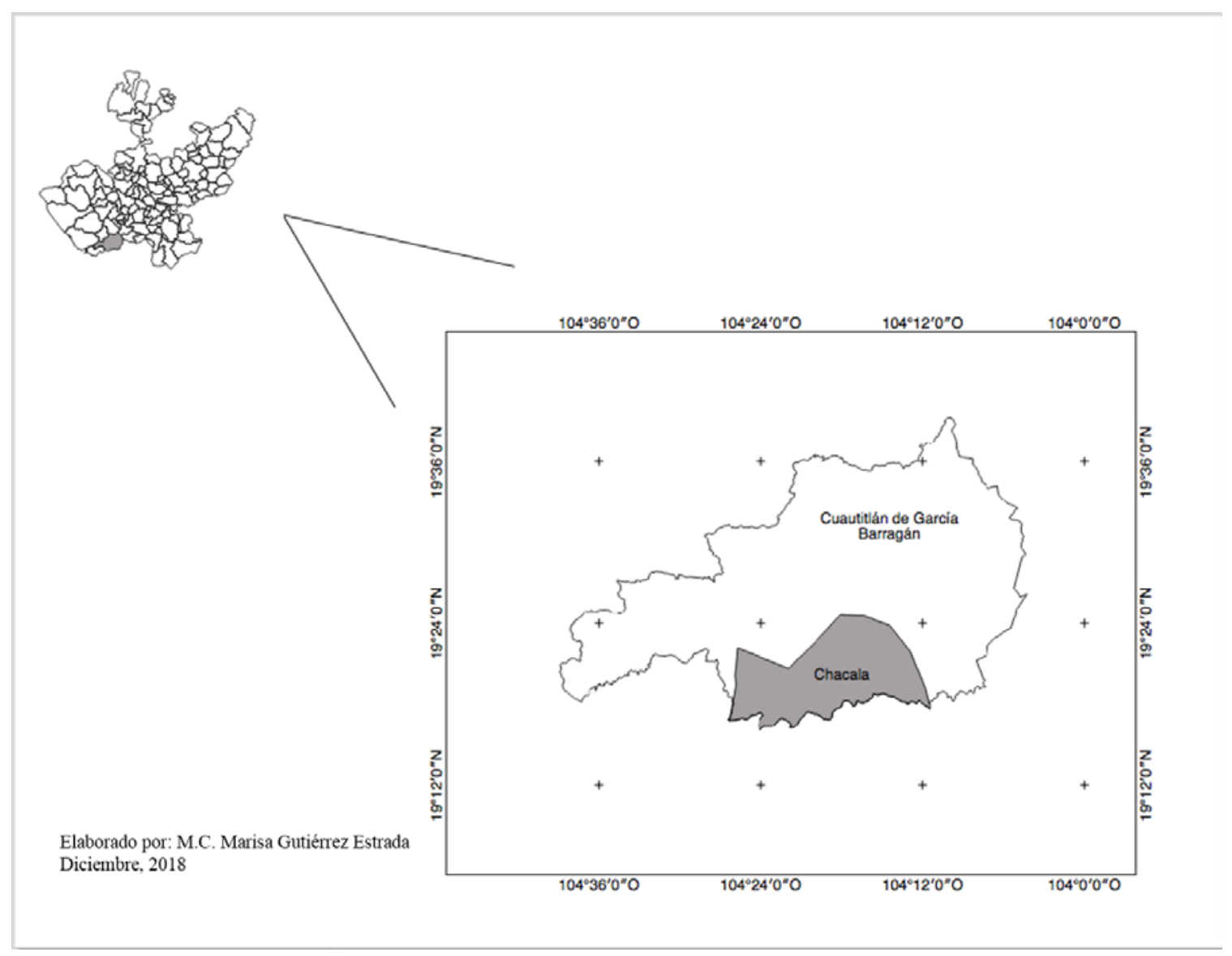

Fuente: GUTIERREZ (2018).

La comunidad cuenta con una delegación, un centro de salud, tiene tiendas para consumo, dos loncherías, una casa ejidal y escuelas (primaria, secundaria y preparatoria). El clima predominante es semiseco y cálido, presenta una temperatura media anual de $23.5^{\circ} \mathrm{C}$, la comunidad cuenta con ríos, arroyos, la mayor parte de su vegetación se compone de fresnos, pinos, cedros, sembradíos de jamaica, maíz, frijol y tomate.

La fuerza principal de la economía de Chacala se centra en la agricultura con alimentos como el frijol, maíz, pepino, jamaica, calabaza que son los principales dentro del consumo cotidiano y que se pueden ofrecer en otras comunidades cercanas. La población tiene conexión económica y laboral con Manzanillo en el estado de Colima, que están interesados en los alimentos que se producen dentro de Chacala, principalmente los chacales de río, y se les facilita hacer las compras del hogar, además de ahorrar, ya que los precios son más bajos dentro del vecino estado de colima.

\section{Propuesta de Desarrollo Turístico}

Con el fin de lograr desarrollo exitoso de un proyecto turístico comunitario, resulta indispensable además de contar con una riqueza biocultural significativa que pueda ser aprovechada de manera sustentable, la capacitación y organización de los pobladores que permita trabajar de manera conjunta y ordenada para alcanzar objetivos comunes que beneficien a todos.

En este contexto, la elaboración de un autodiagnóstico comunitario constituye una manera de determinar la viabilidad de un proyecto y un medio para la obtención de información importante sobre la comunidad, sustentado en la experiencia local pueden surgir ideas y planteamientos concretos para la 
elaboración de proyectos que respondan a sus necesidades o al aprovechamiento de sus atractivos y fortalezas, de tal manera que al ser detectada la problemática o potencial, se pueda empezar a dar propuestas de solución a los problemas o para el aprovechamiento de sus recursos y atractivos, de una manera más organizada.

La presente propuesta se realizó bajo la premisa de revalorizar las actividades socio-culturales y naturales de la comunidad de Chacala, en el municipio de Cuautitlán de García Barragán, Jalisco, México a partir del desarrollo de propuestas de actividades recreativas fundamentadas en el turismo natural y cultural, enfatizando la puesta en valor de la gastronomía regional como un elemento clave en la construcción de la hospitalidad y el desarrollo de la actividad turística basada en el turismo rural, en el cual se desarrollan diversos esquemas para el mejoramiento de la calidad de vida de las personas involucradas, el manejo adecuado de sus recursos y actividades productivas. Dada la importancia de ésta actividad en los diversos procesos sociales y culturales, las comunidades rurales, siendo en su mayoría indígenas participan de manera activa, ya que concentran un legado cultural; se identifica respecto del resto de la población porque habla un idioma distinto a la lengua oficial; y que además tiene usos y costumbres distintas; y cuya organización política, social, cultural y económica se diferencia de los otros sectores sociales, porque se sostiene en sus costumbres (RAMíREZ, 2007: 211-212).

Este proyecto también se desarrolla a partir de la participación comunitaria y la asesoría externa, con el objetivo de generar alternativas económicas en beneficio local, al mismo tiempo que se diversifica la oferta turística regional.

\section{Apuntes metodológicos}

El trabajo de investigación desarrollado es de tipo exploratorio-descriptivo y proponiendose estudiar la potencialidad turística de la Comunidad Indígena de Chacala, para determinar si en conjunto, los elementos físico-geográfico y socioeconómico-cultural, constituyen un potencial de interés, que pueda favorecer el desarrollo de actividades turísticas de bajo impacto o sustentables, en las que se podrían involucrar a las localidades rurales indígenas que se localizan en el municipio, para mejorar su calidad de vida.

Siendo este integrado de las siguientes etapas:

Fase I visita de reconocimiento

- $\quad$ Recorrido e identificación de elementos bioculturales.

- Reunión con las autoridades de la comunidad para hacer el planteamiento del proyecto en sesión ejidal ordinaria, bajo la probación del mismo comisariado ejidal, donde además se realizaron entrevistas informales.

Fase II Intervención social

Presentación y aprobación del proyecto, charla de sensibilización y socialización de objetivos mediante un taller participativo, en el taller se realizó la identificación y evaluación de atractivos, mismos que se constrastaron con el trabajo realizado en la primera fase y que sirvió como complemento ante la percepción y valorización de los diferentes actores municipales

Fase III Identificación de atractivos

- Recorrido por sitios elegidos y levanta miento de información geográfica.

- Evidencia fotográfica.

Fase IV Análisis y evaluación

- Organización y representación de datos, desarrollo de tablas o gráficas.

Fase V Resultados y propuestas

Planteamiento de los resultados obtenidos

A partir de la metodología planteada se buscaron diversos medios para recabar la información y obtener resultados desde la perpectiva de los actores sociales y su percepción directa, del mismo modo se identificó la pertinencia del Diagnóstico Rural Participativo, y que en adelante se hará referencia como (DRP), (ESTELÍ, 2008: 8). En la siguiente 
sección se describe el proceso bajo el cual se llevó a cabo el proceso para la elaboración en la comunidad indígena de Chacala.

\section{Diagnóstico Rural Participativo en la comunidad indígena de Chacala, Cuautitlán de García Barragán, Jalisco}

El DRP tiene como propósito fortalecer capacidades y habilidades de hombres u mujeres que en la práctica están conduciendo procesos de desarrollo desde sus comunidades (ESTELÍ, 2008: 9). A partir de la premisa anterior se plateó el siguiente objetivo:

Objetivo: Obtener de manera directa información primaria en la comunidad de Chacala, con un grupo representativo de sus pobladores, hasta llegar a un autodiagnóstico socioproductivo y del patrimonio natural y cultural.

En el siguiente cuadro se presentan los principales resultados obtenidos, utilizando el taller participativo como principal técnica para recabar información y posteriormente sistematizarla.

Cuadro 1: Herramientas para el taller comunitario.

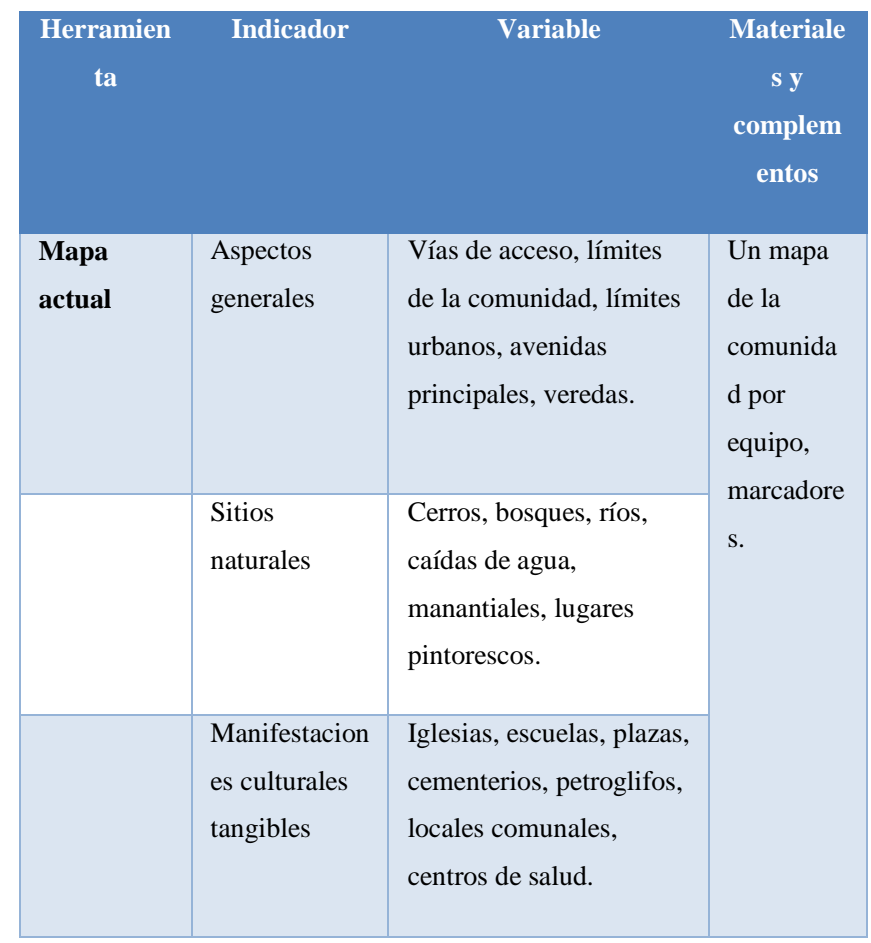

\begin{tabular}{|c|c|c|c|}
\hline & $\begin{array}{l}\text { Actividades } \\
\text { agropecuarias } \\
\text { y pesqueras }\end{array}$ & $\begin{array}{l}\text { Forestales, } \\
\text { agropecuarias, cosechas, } \\
\text { pesqueras. }\end{array}$ & \\
\hline $\begin{array}{l}\text { Calendario } \\
\text { de } \\
\text { actividades }\end{array}$ & $\begin{array}{l}\text { Actividades } \\
\text { continuas o } \\
\text { estacionales. }\end{array}$ & $\begin{array}{l}\text { Forestales, } \\
\text { agropecuarias, } \\
\text { pesqueras, cosechas, } \\
\text { trabajo colectivo. }\end{array}$ & $\begin{array}{l}\text { Formato } \\
\text { en } \\
\text { papelote }\end{array}$ \\
\hline $\begin{array}{l}\text { Diagrama } \\
\text { histórico }\end{array}$ & $\begin{array}{l}\text { Historia de la } \\
\text { comunidad }\end{array}$ & 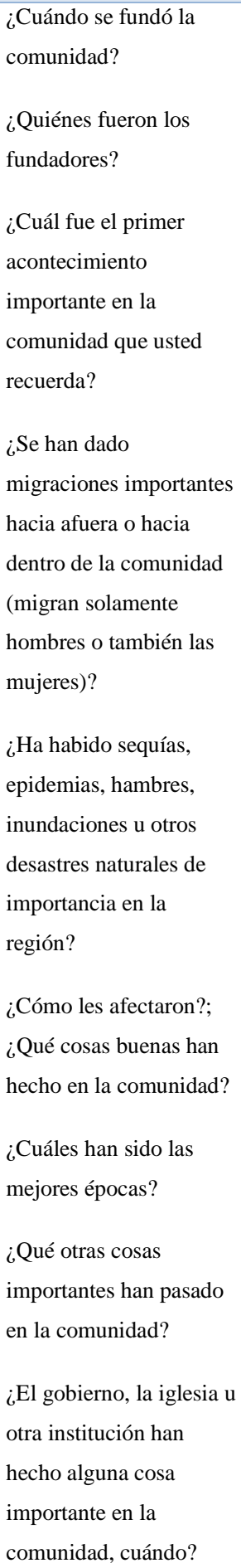 & $\begin{array}{l}\text { Papelote } \\
\text { en } \\
\text { plenaria }\end{array}$ \\
\hline
\end{tabular}




\begin{tabular}{|c|c|c|c|}
\hline $\begin{array}{l}\text { Cuadro } \\
\text { representa } \\
\text { tivo del } \\
\text { patrimonio } \\
\text { inmaterial }\end{array}$ & Folklore & $\begin{array}{l}\text { Leyendas, tradiciones, } \\
\text { música y danza, } \\
\text { artesanía, mitos, } \\
\text { gastronomía, etnológico, } \\
\text { herbolaria y medicina } \\
\text { tradicional }\end{array}$ & $\begin{array}{l}\text { Papelote y } \\
\text { posticks }\end{array}$ \\
\hline & $\begin{array}{l}\text { Acontecimien } \\
\text { tos } \\
\text { programados }\end{array}$ & $\begin{array}{l}\text { Fiestas patronales, } \\
\text { religiosas, carnavales. }\end{array}$ & \\
\hline $\begin{array}{l}\text { Reloj de } 24 \\
\text { horas }\end{array}$ & $\begin{array}{l}\text { Actividades } \\
\text { diarias } \\
\text { comunales }\end{array}$ & Descripción de un día & $\begin{array}{l}\text { Formato } \\
\text { Individual }\end{array}$ \\
\hline $\begin{array}{l}\text { Entrevista } \\
\text { s } \\
\text { semiestruc } \\
\text { turadas }\end{array}$ & $\begin{array}{l}\text { Diversos } \\
\text { temas }\end{array}$ & $\begin{array}{l}\text { Ampliación de la } \\
\text { información }\end{array}$ & $\begin{array}{l}\text { Cuestiona } \\
\text { rios }\end{array}$ \\
\hline
\end{tabular}

Fuente: Elaboración propia a partir del taller participativo.

A partir de la información anterior se determinaron carácteristicas y elementos del patrimonio, cuya importancia es relevante para la planificación de actividades a partir de sus capacidades locales y valorización socioeconómica, socioecológica y socioambiental como ejes principales.

\section{- Chacales}

Para los nahuas, las tradiciones ancestrales tienen gran valor cultural, que se transmite de generación en generación. A continuación, un miembro del consejo de mayores (autoridad comunitaria náhuatl) una de sus más grandes festividades:

"En lo alto de la sierra, desemboca el río Chacala "río de chacales" (por su nombre en náhuatl) es parte fundamental para los pueblos indígenas, proveyendo de agua a las parcelas, de sonidos al viento y vida a los paisajes, mismo que ve nacer al alimento por excelencia de la temporada de aguas (lluvias) "Los Chacales", cuya tradición tiene lugar desde su cuidado, extracción, preparación y consumo.

... Inicia la temporada de aguas, la gente contenta observa el río Chacala, lo verde del campo embellece los paisajes y entre ellos susurran: "falta poco para que lleguen los Chacales, esos de gran tamaño y mejor sabor"...

El plazo ha llegado, es tiempo de que los hombres empiecen a tejer sus chacaleras ${ }^{1}$, sentados en los patios de sus casas, mientras ven la lluvia caer, pensando en el mejor sitio para colocarlas y esperar que el río sea generoso y les deje buena cosecha. Salen por las noches, con chacaleras al hombro, las colocan en un sitio estratégico y esperan pacientemente mientras escuchan la fuerza del río después de la tormenta.

Si fue una noche con éxito, las chacaleras están repletas, chacales de todos tamaños, listos para la fiesta de inicio del temporal, el día en que se da gracias por las cosechas y por las bondades de la sierra y el agua.

Mientras tanto en casa, la mujer prepara la cocina, con las sartas ${ }^{2}$ de tomatillo cosechado de entre la milpa en el temporal de secas y los chiles de sus propias huertas. Huele a festejo, huele a historia, huele a respeto por la vida y la tierra.

Los chacales han sido cuidadosamente preparados, con la receta de los antepasados, los indígenas. Hombres y mujeres ya han hecho su trabajo, es hora de la fiesta.

\footnotetext{
${ }^{1}$ Herramienta hecha de otate, en forma de canasta cónica, que se coloca río abajo y en la cual caen los chacales arrastrados por la corriente del río
} 
El pueblo se reúne, comandados por el consejo de mayores (autoridad comunitaria indígena) se escucha la música de tambores y violines (danza tradicional de Chacala) y se degustan los Chacales, se han esperado todo un año para la cosecha mayor y eso tiene un valor excepcional para ellos.

Todos sonríen, dan gracias al río Chacala "Río de Chacales" en el idioma madre (náhuatl), (GUTIÉRREZ, 2009: 4). ${ }^{3}$

Tlazohcamati miyac (muchas gracias)

Mah xitlacuacan cualli (buen provecho)

Son camarones de río (agua dulce) que poseen un cuerpo comprimido, generalmente robusto se les puede encontrar con varios nombres coloquiales dependiendo de la región tales como; langostinos, chacales, acamallas, cauques o gambas, pertenecen al género Macrobrachium este género es integrado por al menos 238 especies que se distribuyen en la franja tropical y subtropical de todo el mundo, de los cuales 26 son nativos de América y su distribución se comprende desde Baja California en México hasta Perú (VEGA, 2015: 273-274), en México se han identificado 17 especies de las cuales cinco son de importancia económica $M$. carcinus, $M$. olfersi y $M$. achantarus en la costa del Atlántico, y $M$. tenellum y $M$. americanum en la costa del Pacífico (VEGAVILLASANTE et al., 2011a)

Cuadro: Especies del género Macrobrachium en Jalisco.

\section{$\sqrt{ }$ Registro \\ *Probable}

Fuente a partir de VEGA, 2015; 276

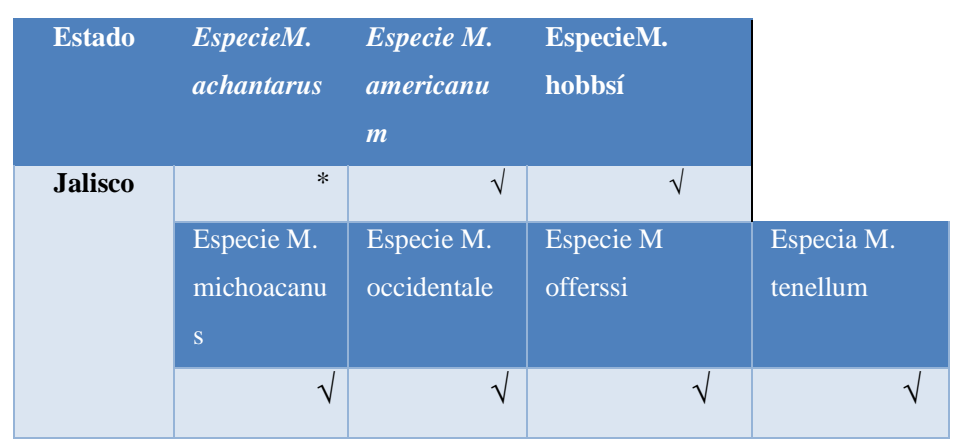

La temporada de chacales es en el temporal de lluvias desde el mes de abril o mayo, y no todas las veces son accesibles, debido a la escases provocada por la contaminación del agua. La venta de chacales sin cocinar es muy demanda y ayuda en la economía de la comunidad, dependiendo del tamaño del chacal implica el precio, entre más grande más caro. Tiene diferentes formas de preparación, como al caldillo, a la diabla y al ajillo, entomatado, entre otras.

\section{- Jamaica}

Rosa de jamaica (hibiscus sabdariffa) de la familia de las Malvaceas, originaria de África fue introducida a México en la época colonial (CID, 2012: 47), es uno los productos que más se cosecha dentro de la comunidad, las personas la utilizan para hacer agua fresca, el proceso de elaboración es fácil, todas las personas dentro y fuera de la comunidad la elaboran. La temporada de cosecha es en junio, y como se seca la jamaica puede ser guardada y se conserva para poderse consumir en cualquier día del año.

\section{Conclusiones}

Como ya se ha mencionado en secciones anteriores, la cocina- está relacionada con la historia, con lo que somos y a lo que pertenecemos-, es decir, con nuestra identidad. MINTZ (2003:28) señala que comer no es una actividad puramente biológica pues los "... alimentos que se comen tienen historias

tienen voz y voto en la toma de decisiones en diversas áreas productivas y sociales comunitarias. Ellos narraron las tradiciones que se llevaban a cabo en la comunidad y que son recuerdos de la infancia vivida. 
asociadas con el pasado de quienes los comen; las técnicas empleadas para encontrar, procesar, preparar, servir y consumir esos alimentos varían culturalmente y tienen sus propias historias. $\mathrm{Y}$ nunca son comidos simplemente; su consumo está condicionado por el significado...". (MINTZ, 2003: 28; CAÑES y TORRES, 2009: 186).

El mayor potencial para desarrollar se detecta en los chacales y la jamaica, alimentos que podrían ser la base del desarrollo de productos gastronómicos que giren entorno a ellos, estos alimentos sin cocinar ya generan un importante ingreso a la comunidad, sin embargo, incursionar en su procesamiento brindaría posibilidades de convertirse en sello identitario que reafirme la hospitalidad de la comunidad.

\section{Referencias}

1 CAMARGO, L.O.L. Os domínios da hospitalidade. Im Dencker, A:F:M; Bueno, M.S.(org) Hospitalidades: cenários e oportunidades. Thomson Leraning, São Paulo. 2003.

2 CANIRAC. Todo sobre la Mesa: Dimesiones de la Insdustría Restaurantera. 2015.

3 CID ORTEGA S \& Guerrero Beltrán J. A. Propiedades de la jamaica (Hibiscus sabdariffa L.) Temas Selectos de Ingeniería de Alimentos 6-2: 47-63. 2012.

4 CAÑEZ, DE LA FUENTE, G.M. y MELÉNDEZ TORRES. La cocina Tradicional Regional como elemento de identidad y desarrollo local: el caso de San Pedro El Saucito, Sonora, México. Revista Estudios sociales, Vol. 17. Spe. México, nov. 2009.

5 CORRAL, M. Reconocen localidades de comunidades indígenas en la región. Retrieved from http://www.radiocosta.com.mx/reconocenlocalidades-de-comunidades-indigenas-en-laregion/. 2015.

6 DARCY VICTOR TETREAULT, C. F.. Jalisco: pueblos indígenas y regiones de alto valor biológico. Espiral, Estudios sobre Estado y Sociedad, 174. 2011.

7 ESTELÍ. Diagnóstico Rural Participativo (DRP) y planificación comunitaria. Instituto de formación permanente, Programa Especial para la Seguridad Alimentaria Nutricional de Nicaragua. 2008.

8 FOX, R. «Reinventing the gastronomic identity of
Croatian tourist destinations». International Journal of Hospitality Management, no 3, 546559. 2007.

9 GUTIÉRREZ, ESTRADA, MARISA. Historias de Vida Locales, realizado en la comunidad indígena de Chacala. Documento técnico. Universidad de Guadalajara, Autlán, Jalisco. 2009.

10 HALL, C. M. Y SHARPLES, L. «The consumption of experiences or the experiences of consumption? An introduction to the tourism of taste» en Food tourism around the world: Development, management and markets (Hall, C. M., Sharples, E., Mitchell, R., Macionis, N. y Cambourne, B., Coord.). Oxford, Edit. Butterworth-Heinemann, 1-24. 2003.

11 HERNÁNDEZ MOGOLLÓN, JOSÉ MANUEL, DICLEMENTE ELIDE, LÓPEZ GUZMÁN TOMÁS. El Turismo Gastronómico como Experiencia Cultural: EL Caso Práctico de la Ciudad de Cáceres (España). Boletín de la Asociación de Geógrafos Españoles № 68, págs. 407-427. 2015.

12 INEGI. Censo Economico 2014.

13 KIVELA, J. Y CROTTS, J. Tourism and gastronomy: gastronomy's influence on how tourists experience a destination». Journal of Hospitality \& Tourism Research, no 3, 354-377. 2006.

14 KPMG. La Gastronomía en la Cocina Española: Impacto económico de los sectores asociados 2019.

15 LASHLEY, C. Para um entendimiento teórico. In Lashley, C y Morrison, A. (org). Em busca da Hospitalidades: perspectivas para um mundo globalizado. Manole, São Paulo: 1-24. 2004.

16 OCDE Comida y la experiencia turística: La OCDE-Corea del taller, Los estudios de la OCDE sobre Turismo, OECD Publishing. 2012.

17 OMT. Panoroma OMT del turismo internacional. Edición 2018.

18 PÉREZ SÁNCHEZ MÓNICA Y CISNEROS MÚJICA WALTER DANIEL. El Lado Cultural del Patrimonio Gastronómico Mexicano, Culinaria, Revista Virtual Gastronomíca, № 3 Nueva Epoca, Julio/diciembre UAEM. 2012.

19 RAMÍREZ VELÁZQUEZ CÉSAR AUGUSTO. Las comunidades indígenas como usuarios de la información. Investigación Bibliotecológica, Vol. 21, Núm. 43 Jul/diciembre, México 2007.

20 TELFER, D. J. Y WALL, G. «Strengthening backward economic linkages: Local food purchasing by three indonesian hotels». Tourism Geographies, no 4, 421-447. 2000. 
21 TORRES BERNIER, E. Del turista que se alimenta al que busca comida. Reflexiones sobre las relaciones entre gastronomia y turismo. En Gatsronomía y Turismo, Vol. 2: Cultura al Plato. Ciet, Buenos Aires, pp. 305. 316. 2003.

22 UNESCO La cocina tradicional mexicana, cultura comunitaria, ancestral y viva - El paradigma de Michoacán. México: UNESCO. Recuperado de https://ich.unesco.org/es/RL/la-cocinatradicional-mexicana-cultura-comunitariaancestral-y-viva-el-paradigma-de-michoacan00400. S.f.

23 VARGAS AVALOS ABEL. Cuautitlán de García de Barragán, Historia, Ayuntamiento Constitucional 2012-2015. 2015

24 VEGA-VILLASANTE, F., E. MARTÍNEZ-LÓPEZ, L. ESPINOSA-CHAURAND, M. Cortés- Lara y $\mathrm{H}$. Nolasco-Soria, «Crecimiento y supervivencia del langostino (Macrobrachium tenellum) en cultivos experimentales de verano y otoño en la costa tropical del Pacífico mexicano», Tropical and Subtropical Agroecosystems, 14 (2011b), pp. 581-588.

VEGA-VILLASANTE F., García-Guerrero M.U., Cortés Jacinto E., Yamasaki-Granados S., Montoya-Martínez C.E., Vargas-Ceballos M.A., Chong-Carrillo O., Rubio-Padilla M.A., GuzmanArroyo M., Carrillo-Farnés O.V., EspinosaChaurand L.D. \& Nolasco-Soria H.G. Los camarones de agua dulce del género Macrobrachium: bilogía, ecología y explotación. In: Temas Sobre Investigaciones Costeras (ed. by J.L. Cifuentes-Lemus \& F.G. Cupul-Magaña), pp. 273-315. Universidad de Guadalajara, Guadalajara, Jalisco, Mexico. 2014. 